\title{
TEMPERAMENTO E DESENVOLVIMENTO DA CRIANÇA: REVISÃO SISTEMÁTICA DA LITERATURA ${ }^{1}$
}

\author{
Vivian Caroline Klein* \\ Maria Beatriz Martins Linhares"
}

\begin{abstract}
RESUMO. O temperamento é uma variável da pessoa que interage com variáveis ambientais e pode assim influenciar as trajetórias de desenvolvimento da criança. O presente estudo teve por objetivo revisar de forma sistemática estudos empíricos, publicados de 2001 a 2006, sobre o tema temperamento e sua relação com o desenvolvimento da criança. Foram selecionados 50 estudos empíricos indexados nas bases de dados PsycINFO, MEDLINE e LILACS por meio de levantamento bibliográfico sistematizado.Verificou-se que a abordagem teórico-conceitual mais adotada pelos estudos foi a abordagem psicobiológica de Rothbart. O temperamento foi avaliado predominantemente por meio de questionários respondidos pelas mães. Os resultados dos estudos indicaram que crianças com o temperamento caracterizado por alto nível de humor negativo, medo, timidez e raiva encontram-se, do ponto de vista do desenvolvimento, suscetíveis de apresentar problemas de desenvolvimento. O temperamento com alto nível de controle com esforço, que indica a capacidade de superar tendências comportamentais e motivacionais dirigidas pelo afeto e reprogramar seu comportamento em situações de conflito de interesses associa-se a resultados desenvolvimentais positivos.
\end{abstract}

Palavras-chave: Temperamento; desenvolvimento infantil; comportamento.

\section{TEMPERAMENT AND CHILD DEVELOPMENT: SYSTEMATIC REVIEW OF THE LITERATURE}

\begin{abstract}
Temperament is a personal variable which interacts with environmental variables and therefore may have an influence on child developmental pathways. The purpose of the present study was to review systematically the empirical studies published from 2001 to 2006 about temperament and its relationship to child development. Fifty empirical studies indexed in the data bases PsycInfo, MEDLINE and LILACS were selected through systematic bibliographic review. It was found that the most frequently used theoretical approach for studying temperament was the Rothbart's psychobiological approach. Temperament was assessed predominantly through questionnaires answered by mothers. The findings of the studies showed that children with temperament characterized by high levels of negative mood, fear, shyness and anger are vulnerable for exhibiting developmental problems. Temperament with high levels of effortful control, which indicates the ability to suppress behavioral and motivational trends guided by the affect and to reprogram the behavior in situations of conflict of interests, is associated to adaptive developmental outcomes.
\end{abstract}

Key words: Temperament; childhood development; behavior.

\section{TEMPERAMENTO Y DESARROLLO INFANTIL: REVISIÓN SISTEMÁTICA DE LA LITERATURA}

RESUMEN. El temperamento es una variable de carácter personal que interactúa con las variables ambientales y por lo tanto puede tener una influencia en el desarrollo infantil. El objetivo de este estudio fue revisar de forma sistemática los estudios empíricos publicados desde 2001 hasta 2006 sobre el temperamento y su relación con el desarrollo del niño. Cincuenta estudios empíricos indexados en las bases de datos PsycINFO, MEDLINE y LILACS se seleccionaron mediante una revisión bibliográfica sistemática. Se encontró que el enfoque más utilizado para el estudio teórico temperamento fue el Rothbart `s enfoque psicobiológico. El temperamento se evaluó principalmente a través de cuestionarios respondidos por las madres. Los resultados de los estudios mostró que los niños con temperamento con altos niveles de estado de ánimo negativo, miedo, timidez e ira son vulnerables para la exposición de problemas de desarrollo. Temperamento con altos niveles de control con

\footnotetext{
Apoio: FAPESP e CNPq.

Doutora em Ciências (Saúde Mental). Professora do Departamento de Neurociências e Ciências do Comportamento da Faculdade de Medicina de Ribeirão Preto, Universidade de São Paulo.

\# Doutora em Psicologia Experimental, IP-USP. Professora Associada (Livre-Docente) do Departamento de Neurociências e Ciências do Comportamento da Faculdade de Medicina de Ribeirão Preto, Universidade de São Paulo.
} 
esfuerzo, lo que indica la capacidad de suprimir las tendencias de comportamiento y de motivación guiada por el afecto y volver a programar el comportamiento en situaciones de conflicto de intereses, se asocia a los resultados del desarrollo adaptativo.

Palabras-clave: Temperamento; desarrollo infantil; comportamiento.

O temperamento é concebido como um conjunto de diferenças individuais estáveis de forte base genética e neurobiológica que aparecem a partir do nascimento (Fox, Henderson, Rubin, Calkins, \& Schmidt, 2001). Os estudos diferem na ênfase atribuída ao papel dos processos emocionais, componentes estilísticos e processos atencionais na origem do temperamento (Mervielde, de Clercq, de Fruyt, \& Van Leeuwen, 2006).

No estudo do temperamento da criança aparecem quatro principais abordagens teórico-metodológicas, propostas, respectivamente, por: Thomas e Chess (1977); Buss e Plomin (1984); Rothbart (1981) e Kagan (1998). Essas abordagens refletem diferentes concepções e procedimentos de avaliação do temperamento.

Na abordagem de Thomas e Chess (1977) o temperamento é concebido como as diferenças individuais no componente estilístico do temperamento, focalizando a maneira pela qual este se manifesta, sua razão e suas características observáveis por meio do comportamento (Muris \& Ollendick, 2005). Esses autores propuseram um sistema de nove categorias: nível de atividade, ritmo, aproximação ou retraimento, adaptabilidade, limiar de responsividade, intensidade de reação, qualidade de humor, distractibilidade e período de atenção e persistência. Essas categorias deram origem às seguintes classificações de tipos de temperamento: temperamento fácil, caracterizado por regularidade nas funções biológicas, respostas de aproximação positiva a estímulos novos, alta adaptabilidade a mudança, assim como intensidade de humor de leve a moderada e preponderantemente positiva; temperamento difícil, caracterizado por sinais de irregularidade nas funções biológicas, respostas de retraimento negativo a novos estímulos, não adaptação ou adaptação lenta a mudanças e expressões de humor intensas, frequentemente negativas; temperamento lento para reagir, caracterizado pela combinação de respostas negativas a estímulos novos com adaptabilidade lenta após contatos repetidos.

$\mathrm{Na}$ abordagem proposta por Buss e Plomin (1984), o temperamento é definido como um conjunto de traços de personalidade hereditários que aparecem cedo no desenvolvimento. $\mathrm{O}$ temperamento é constituído por três traços específicos: 1) emocionalidade, caracterizada por instabilidade psicológica e propensão a experimentar sentimento de medo, raiva e tristeza; 2) atividade, referente a características como tempo, vigor e resistência, que pode ser medidos pela frequência e amplitude da fala e do movimento e pelo deslocamento dos movimentos corporais e duração de comportamento agitado; 3) sociabilidade, que se refere a traços como tendência de afiliação e responsividade aos outros, podendo ser avaliada pela preferência por estar com os outros e pela necessidade de compartilhar atividades e receber atenção como resultado da interação social.

A abordagem desenvolvida por Rothbart (1981) modificou a concepção do temperamento, passando de um modelo estilístico para um modelo psicobiológico (Goldsmith, 1983). Nesta abordagem, o temperamento é entendido como diferenças individuais com base constitucional na reatividade e autorregulação, influenciadas ao longo do tempo pela hereditariedade, maturação e experiência. A constituição é definida como a composição relativamente biológica do indivíduo, influenciada através do tempo pela hereditariedade, maturação e experiência. A reatividade é entendida como característica da responsividade individual a mudanças de estimulação apresentada em diversos níveis (comportamental, autonômico e neuroendócrino) e por meio de parâmetros de latência, tempo de aumento, intensidade máxima e tempo de recuperação da reação. A autorregulação é definida como processos que modulam essa reatividade, incluindo abordagem/retraimento comportamental, controle inibitório e tentativa de controle da atenção (Putnam, Gartstein, \& Rothbart, 2006).

A abordagem psicobiológica de Rotbarth difere das abordagens anteriores, pois entende que as diferenças individuais vão além dos estilos comportamentais, incluindo as diferenças no funcionamento psicofisiológico, a susceptibilidade às emoções primárias (Goldsmith et al., 1987) e a autorregulação da atenção. Além disso, compreende uma perspectiva desenvolvimental, ao avaliar o temperamento em diferentes fases do ciclo vital.

$\mathrm{Na}$ abordagem proposta por Kagan, Reznick e Snidman (1987), o temperamento é entendido como um padrão de sequências de comportamentos que se relacionam a um padrão de reações fisiológicas 
particulares inatas em resposta a um estímulo específico. As dimensões do temperamento são estudadas de acordo com dois polos extremos de diferentes dimensões, tais como temperamento inibido ou desinibido, afetividade positiva ou negativa, entre outras.

O estudo do temperamento evidencia processos existentes no início da vida a partir dos quais se desenvolvem as adaptações sociais às condições ambientais. A detecção precoce de determinadas características de temperamento que podem influenciar o desenvolvimento da criança contribui tanto para a promoção do desenvolvimento quanto para a prevenção de problemas de saúde mental (Klein \& Linhares, 2007).

$\mathrm{Na}$ literatura científica brasileira identifica-se uma lacuna em relação a publicações que sistematicamente analisem a produção empírica atual sobre a relação entre o temperamento e o desenvolvimento da criança.

\section{OBJETIVO}

O presente estudo teve como objetivo realizar uma revisão sistemática da literatura sobre o tema temperamento e sua relação com o desenvolvimento da criança, mediante a análise dos estudos empíricos publicados em periódicos indexados.

\section{MÉTODO}

O levantamento bibliográfico foi realizado nas bases de dados MEDLINE e PsycINFO por meio das seguintes palavras-chaves: temperament AND child development OR developmental outcomes e suas correspondentes na Língua Portuguesa no LILACS. Foram definidos os seguintes critérios de inclusão: o artigo ser empírico e publicado em inglês, português ou espanhol; o estudo ter sido realizado com seres humanos a partir do nascimento até os 12 anos de idade; e o artigo ter sido publicado no período de janeiro de 2001 a janeiro de 2006 e ter tido como objetivo estudar o temperamento e sua relação com o desenvolvimento da criança. Foram excluídos: estudos com objetivo exclusivamente psicométrico; estudos que avaliavam os efeitos de medicação/drogas/intervenção no temperamento; e estudos que avaliavam o efeito do temperamento da criança nas características parentais.

Por meio das palavras-chave foram identificados 98 artigos nas bases de dados especificadas anteriormente, e de acordo com os critérios de inclusão e exclusão, foram selecionados para análise 50 artigos.

$\mathrm{Na}$ análise dos artigos foram tratados os seguintes itens: abordagens teórico-conceituais que fundamentaram o estudo do temperamento; procedimentos e instrumentos adotados para avaliação do temperamento; características das amostras estudadas e os principais resultados dos estudos.

\section{RESULTADOS}

\section{Abordagens teóricas que fundamentaram os estudos sobre temperamento}

A abordagem psicobiológica, proposta por Rothbart, foi utilizada em 21 estudos (42\%); em 13 estudos $(26 \%)$ foi adotada a abordagem proposta por Thomas e Chess; em cinco estudos (10\%) foi utilizada a abordagem proposta por Buss e Plomin; e em apenas três estudos (6\%) foi adotada a abordagem observacional de Kagan. Seis estudos utilizaram abordagens combinadas, dos quais dois combinaram as abordagens de Rothbart e de Thomas e Chess, dois combinaram as abordagens de Rothbart e Kagan e dois estudos combinaram as abordagens de Rothbart, de Buss e Plomin e Kagan. Ao todo, 27 estudos (54\%) tiveram como base a abordagem psicobiológica do temperamento de Rotbarth, revelando uma tendência crescente na literatura ao uso desta abordagem relativamente às abordagens estilísticas do temperamento.

\section{Procedimentos de avaliação do temperamento e instrumentos mais utilizados}

Com relação aos procedimentos de avaliação do temperamento, foram utilizadas duas principais formas de avaliação. Os questionários foram os procedimentos de avaliação mais utilizados, sendo empregados em $88 \%$ dos estudos. Com relação aos respondentes dos questionários, $81 \%$ dos estudos utilizaram como respondentes as figuras parentais das crianças, com predomínio das mães. Apenas 10\% dos estudos utilizaram como respondentes os professores das crianças. Em quatro estudos (8\%) houve a realização de questionários com mais de um informante (pai e mãe ou pais e professores).

A observação sistemática em situação experimental ou ambiente natural foi o segundo procedimento mais utilizado para avaliação do temperamento, ocorrendo em 13 estudos (26\%). Dos estudos observacionais, $14 \%$ combinaram o procedimento de observação com a aplicação de questionários. 
Com relação aos instrumentos de avaliação do temperamento, identificou-se que os questionários mais utilizados pelos estudos foram o $C B Q$-Child Behavior Questionnaire (22\%), para crianças de 3 a 7 anos de idade, e o IBQ-Infant Behavior Questionnaire (20\%), para crianças de 3 a 12 meses de idade, ambos derivados conceitualmente da abordagem de Rothbart.

\section{Características das amostras estudadas}

Em $44 \%$ dos estudos o temperamento foi avaliado na fase do primeiro ano de vida, e em $44 \%$ deles, no quarto ano de vida da criança. As amostras dos estudos eram predominantemente não clínicas (66\%), constituídas de crianças com desenvolvimento típico sem risco estabelecido e sem referência para atendimento clínico por problemas de desenvolvimento ou comportamento. Por outro lado, $40 \%$ dos estudos selecionaram crianças pertencentes a grupos de risco para problemas de desenvolvimento, dos tipos crianças com vulnerabilidade biológica neonatal (16\%), crianças em situação de pobreza (8\%) e crianças em risco direto para psicopatologias devido a diagnóstico de psicopatologias dos pais (transtorno bipolar) ou irmãos (autismo) (4\%). Apenas 4\% dos estudos foram realizados com irmãos gêmeos, a fim de verificar a influência da hereditariedade no temperamento.

Principais resultados dos estudos

Os resultados serão apresentados tendo-se como enfoque os principais achados estatisticamente significativos que demonstraram a relação entre dimensões do temperamento e diferentes fases do desenvolvimento da criança. Serão apresentados também os resultados dos estudos que evidenciaram os correlatos fisiológicos do temperamento.

\section{Temperamento e afetividade}

Em relação ao constructo do apego, Ispa, Fine e Thornburg (2002) encontraram que medo foi relacionado a apego inseguro no primeiro ano de vida, mas apenas para os bebês cujas mães tinham baixo nível de reação ao estresse. No segundo ano de vida da criança, os autores identificaram que altos escores na dimensão medo foram preditores de apego seguro. Outro estudo identificou que, aos 22 meses, crianças com apego classificado como "inativo e autônomo" apresentaram maiores escores na dimensão Inibição comportamental do que as crianças com apego classificado como "de contato mais íntimo e com foco na mãe" (Schieche \& Spangler, 2005). O Medo, avaliado aos 22, 33 e 45 meses de idade em situação não familiar estressante também foi preditor de culpa em cada uma das três idades avaliadas (Kochanska, Gross, Lin, \& Nichols, 2002).

Em relação ao fator Afetividade negativa, as crianças pré-escolares com maiores escores neste fator apresentavam hipersensibilidade na percepção de falhas relacionadas ao seu desempenho em situação de resolução de problemas, ou seja, produziam uma resposta de aflição exacerbada diante da percepção de um erro na tarefa proposta (Luciana, Gunnar, Davis, Nelson \& Donzella, 2005).

No estudo prospectivo-longitudinal de Weinfield, Whaley e Egeland (2004), um grupo de crianças foi acompanhado do nascimento aos 19 anos de idade com foco nos aspectos apego e no temperamento. Verificou-se que participantes com apego seguro e consistente ao longo do tempo foram avaliados por suas mães como de menor limiar de responsividade, menor nível de atividade e de maior persistência de atenção, em comparação aos participantes com apego seguro e posteriormente inseguro. Menor escore no limiar de responsividade indicava que o bebê precisava de menos estimulação para produzir uma resposta, enquanto maior escore em persistência de atenção indicava que o bebê poderia sustentar uma atividade por um longo tempo.

As dimensões raiva e consolabilidade em bebês no primeiro ano de vida foram associadas ao comportamento de cuidado de irmãos na idade préescolar, pois os bebês que apresentaram maiores escores nestas dimensões recebiam mais cuidado por parte de seus irmãos (Volling, Herrera, \& Poris, 2004). No primeiro ano de vida da criança, altos escores na dimensão raiva foram preditores de ciúmes de irmãos em relação à sua mãe (Volling, McElwain \& Miller, 2002).

Em contrapartida, altos escores em controle com esforço foram preditores de melhor autorregulação comportamental em crianças pré-escolares, expressa por meio da capacidade de demonstração de afeto positivo em situações em que estas receberam presentes desejados, como um brinquedo, e presentes indesejados, como, por exemplo, uma planta (Kieras, Tobin, Graziano \& Rothbart, 2005).

\section{Temperamento e socialização}

No tocante à socialização das crianças, temperamento difícil predisse denominações negativas indicativas de rejeição pelos pares na idade pré-escolar (Szewczyk-Sokolowski, Bost \& Wainwright, 2005). Por outro lado, escores mais altos nas dimensões impulsividade e capacidade para se confortar foram preditores de denominações positivas nesta mesma 
idade, ou seja, acolhimento por parte dos colegas (Gleason, Gower, Hohmann \& Gleason, 2005).

A dimensão de temperamento timidez foi mais consistentemente associada ao comportamento social das crianças pré-escolares, de forma que, quanto menor a timidez, tanto maior a disposição física e relacional, a sociabilidade e o comportamento prósocial da criança (Russel, Hart, Robinson, \& Olsen, 2003). Em outro estudo com crianças pré-escolares, timidez também foi associada à socialização, de modo que as crianças avaliadas como sociáveis apresentavam menores escores em timidez do que as crianças categorizadas como reticentes e solitárias (Henderson, Marshall, Fox, \& Rubin, 2004).

Altos escores em controle com esforço, fator do temperamento que possibilita ao indivíduo suprimir tendências comportamentais e motivacionais dirigidas pelo afeto e reprogramar seu comportamento em situações de conflito de interesses, foram preditores de melhor desenvolvimento afetivo-social em crianças pré-escolares, expresso por meio de menor nível de agressividade com os pares e, consequentemente, menor nível de rejeição por parte destes (Gunnar, Sebanc, Tout, Donzella, \& van-Dulmen, 2003).

\section{Temperamento, cognição e linguagem}

Os escores na dimensão do temperamento medo no primeiro ano de vida foram associados ao desenvolvimento cognitivo da criança, de forma que menores escores foram identificados em crianças que apresentavam melhor desempenho de habituação cognitiva (Rieser-Danner, 2003). Na mesma direção, escores mais altos na dimensão aproximação foram preditores de melhor desempenho da memória operativa e controle inibitório da criança na fase préescolar durante a realização de uma tarefa (Wolfe \& Bell, 2004).

Controle com esforço foi preditor de melhor desenvolvimento dos mecanismos atencionais de crianças pré-escolares em condição de pobreza, ou seja, de maior acurácia nas redes executiva, de orientação e de vigilância da atenção (Chang \& Burns, 2005).

Por outro lado, em uma amostra de bebês vulneráveis nascidos pequenos em relação à idade gestacional que apresentaram maiores escores na dimensão reatividade negativa aos três meses apresentaram pior desempenho cognitivo aos seis meses de vida em comparação aos bebês nascidos na mesma condição que apresentavam temperamento menos reativo (Gorman, Lourie, \& Choudhury, 2001).

Aos quatro anos, crianças com maiores escores em timidez apresentaram pior desempenho nas habilidades expressivas e receptivas de linguagem do que crianças que apresentaram baixos escores (Spere, Schmidt, Theall-Honney, \& Martin-Chang, 2004).

\section{Temperamento, problemas de comportamento e} psicopatologia

Algumas categorias e dimensões do temperamento foram associadas a problemas de comportamento da criança e outras psicopatologias. Verificou-se que maiores escores em temperamento difícil aos dois anos de idade foram preditores de problemas de comportamento aos sete anos em crianças nascidas prematuras (Saylor, Boyce, \& Price, 2003).

$\mathrm{Na}$ idade pré-escolar, temperamento difícil foi identificado como uma variável mediadora da relação entre conflito familiar e problema de comportamento externalizante na fase escolar. Em crianças com temperamento difícil durante os anos pré-escolares, conflito familiar predisse problemas de externalização durante os anos escolares; por outro lado, esta relação não foi encontrada no grupo de crianças com temperamento fácil (Ramos, Guerin, Gottfried, Bathurst, \& Oliver, 2005).

Aos três anos de idade, crianças prematuras com problemas de comportamento externalizantes e internalizantes apresentavam maiores escores em emocionalidade negativa do que aquelas sem problemas de comportamento. Medo foi preditor de problemas internalizantes, ao passo que raiva e nível de atividade foram preditores de problemas externalizantes nesta idade (Blair, 2002).

As dimensões flexibilidade, humor positivo $\mathrm{e}$ orientação para tarefa apresentaram menores escores em um grupo de filhos de pais com transtorno bipolar em comparação ao grupo de filhos de pais sem este diagnóstico (Chang, Blasey, Ketter, \& Steiner, 2003). Maiores escores em duração de orientação da atenção, avaliados aos seis meses de vida, foram identificados em crianças que apresentaram diagnóstico de autismo aos dois anos em comparação a crianças que não apresentaram o diagnóstico. Aos dois anos, as primeiras apresentaram menores escores em transferência de atenção, nível de atividade, antecipação positiva e controle inibitório em comparação com as segundas (Zwaigenbaum et al., 2005).

\section{Temperamento e correlatos fisiológicos}

Alguns estudos identificaram a relação entre temperamento e correlatos fisiológicos. No estudo de Doussard-Roosevelt, Montgomery e Porges (2003), a regulação do controle vagal cardíaco foi relacionada 
ao temperamento da criança aos cinco anos de idade. Menores taxas de período cardíaco basal, que indicam maior frequência cardíaca, foram preditoras de maiores escores em timidez. Além disso, diante de uma tarefa estruturada para eliciar afeto negativo, menor atividade da frequência cardíaca predisse maiores escores de sociabilidade. Esta dimensão do temperamento, por sua vez, foi também predita por maior arritmia sinorrespiratória basal, ou seja, maior variabilidade da frequência cardíaca com a respiração.

Raiva, por sua vez, foi associada a um indicador de reatividade fisiológica ante uma situação potencialmente ameaçadora para a criança aos 15 meses de idade, de modo que maiores escores foram associados a um maior aumento de nível de cortisol (van Bakel \& Riksen- Walraven, 2004).

O controle com esforço foi associado ao nível de cortisol salivar (indicador de regulação fisiológica e estresse), que foi avaliado ao longo do dia, em crianças pré-escolares. Maiores escores neste fator do temperamento foram preditores de menores níveis de produção de cortisol salivar (hormônio do estresse), que indicam padrões mais amadurecidos de regulação (Watamura, Donzella, Kertes, \& Gunnar, 2004).

Maiores escores em extroversão foram associados a um menor aumento no nível de cortisol salivar ao longo do dia na primeira semana de aula aos seis anos de idade da criança, o que significa maior regulação comportamental (Bruce, Davis \& Gunnar, 2002).

\section{DISCUSSÃO}

O presente estudo de revisão da literatura identificou que o tema temperamento $e$ desenvolvimento da criança tem sido amplamente estudado. Apesar de os critérios de inclusão terem considerado crianças de zero a doze anos, a maior parte dos estudos analisados concentrou as avaliações de temperamento na fase de um a quatro anos de idade. Isto revela o foco de estudo desta variável nos primeiros anos de vida, que correspondem ao período de maior desenvolvimento cerebral e são marcados por grande impacto nas variáveis biológicas no desenvolvimento (Couperus \& Nelson, 2006).

A análise dos critérios de composição das amostras dos estudos revelou que grande parte dos estudos selecionou amostras não clínicas, ou seja, amostras de crianças não previamente identificadas como em risco de apresentar problemas de desenvolvimento. Isto pode ser interpretado como reflexo dos esforços em compreender o temperamento e sua relação com processos de desenvolvimento típico ou normativo.
Tomando como base a metodologia empregada nos estudos, destacou-se o referencial psicobiológico proposto por Rothbart (1981), o qual entende o temperamento como passível de evolução ao longo da trajetória de desenvolvimento, influenciado tanto pela hereditariedade quanto pela experiência e expresso em termos de reatividade e autorregulação.

A análise dos procedimentos utilizados para avaliação do temperamento revelou o uso predominante de questionários, respondidos, em sua maioria, pelas mães. $\mathrm{O}$ relato dos pais para avaliação do temperamento tem como vantagem o fato de estes verem seus filhos em uma ampla variedade de situações, ética e logisticamente impossíveis de serem recriadas em situação artificial de laboratório. A validade convergente dos questionários de avaliação de temperamento respondidos pelos pais com as escalas de observação sistemática foi previamente constatada, constituindo uma medida objetiva substancial nestes instrumentos, que permitem englobar diferentes fatores e dimensões (Rothbart \& Bates, 1998).

Por outro lado, as observações diretas da criança para avaliar o temperamento em situação de laboratório têm como vantagem serem mais objetivas do que os questionários quando os avaliadores estão bem treinados para minimizar a interação entre criança e avaliador; no entanto, as situações de observação do temperamento têm como limitação a impossibilidade de avaliar múltiplas dimensões simultaneamente (Kagan, 1998).

Desta forma, estudos que combinam relato parental com observação do comportamento, bem como estudos que combinam relato de mais de um informante conhecedor da criança, apresentam vantagens metodológicas em relação aos estudos que adotam apenas uma medida única de temperamento, com base em um único avaliador.

Os questionários mais utilizados são aqueles construídos com base no referencial psicobiológico de Rothbart, o que é um reflexo da predominância da abordagem desta autora nos estudos analisados. Estes questionários avançam no sentido de descreverem comportamentos das crianças em diversas situações específicas e solicitarem julgamentos de frequência de emissão de tais comportamentos em um período de tempo recente, permitindo maior confiabilidade do relato dos pais. Os questionários IBQ e CBQ encontram-se traduzidos para o Português do Brasil por Klein, Putnam e Linhares (2009) e disponíveis para o uso em pesquisa.

Os achados da revisão mostraram que o temperamento difícil foi associado a problemas de 
socialização (Szewczyk-Sokolowski et al., 2005) e de comportamento (Ramos et al., 2005; Saylor et al., 2003), assim como emocionalidade negativa foi associada a problemas de comportamento (Blair, 2002).

Afetividade negativa, por sua vez, foi relacionada ao pior desempenho cognitivo (Gorman et al., 2001) e à hipersensibilidade da criança diante da percepção de falhas cognitivas (Luciana et al., 2005). A dimensão de temperamento medo foi associada a problemas internalizantes (Blair, 2002), apego inseguro (Ispa et al., 2002), culpa (Kochanska et al., 2002) e pior desempenho de habituação cognitiva (Rieser- Danner, 2003). A dimensão raiva, por sua vez, foi associada a problemas externalizantes (Blair, 2002), ciúmes (Volling et al., 2002) e à maior dificuldade de regulação fisiológica expressa por meio de alta concentração do hormônio indicativo de estresse, o cortisol (van Bakel \& Riksen- Walraven, 2004). A dimensão timidez foi relacionada a menos agressão com os pares (Russel et al. 2003), menor socialização (Henderson et al., 2004; Russel et al. 2003), pior desempenho nas habilidades expressivas e receptivas de linguagem (Spere et al., 2004) e maior dificuldade de regulação fisiológica, expressa por maior reatividade de frequência cardíaca (DoussardRoosevelt et al., 2003).

Por outro lado, o controle com esforço foi associado a melhor autorregulação fisiológica (Watamura et al., 2004), melhor desenvolvimento da atenção (Chang \& Burns, 2005), maior capacidade de regulação do comportamento de acordo com as normas sociais (Kieras et al., 2005) e melhor socialização (Gunnar et al., 2003), assim como ao comportamento de cuidado de irmãos (Volling et al., 2004).

Os estudos que identificaram correlatos fisiológicos de dimensões do temperamento, ou seja, que relacionaram a atividade autonômica (período cardíaco e arritmia sinorrespiratória) e neuroendócrina (produção de cortisol salivar) a dimensões como controle com esforço, medo e timidez, contribuem para a compreensão dos mecanismos subjacentes dos comportamentos observados, fortalecendo o referencial teórico-conceitual psicobiológico de Rothbart.

Os estudos sugerem que as crianças com o temperamento caracterizado como irregular, com humor negativo, com medo, timidez e raiva encontram-se suscetíveis de apresentar problemas de desenvolvimento. As habilidades relacionadas ao controle com esforço, que indica capacidade de regulação fisiológica e comportamental, controlando e inibindo um comportamento dominante a favor de um comportamento subdominante, associam-se a resultados desenvolvimentais positivos.

\section{CONSIDERAÇÕES FINAIS}

A presente revisão sistemática da literatura contribuiu no sentido de identificar a abordagem teórico-conceitual mais adotada pelos estudos empíricos para estudar o temperamento, as metodologias utilizadas para a avaliação deste e as características utilizadas para a seleção das amostras dos estudos. Além disso, permitiu a compilação dos principais resultados empíricos sobre a relação do temperamento com o desenvolvimento da criança. Os achados dos estudos demonstraram que o temperamento se relaciona a diferentes áreas do desenvolvimento humano, o que justifica a sua avaliação em estudos que têm por objetivo identificar os processos de inter-relação entre variáveis individuais e ambientais que resultam em uma dada trajetória de desenvolvimento. Pesquisas recentes têm avançado ao investigar correlatos fisiológicos do temperamento, consolidando o enfoque psicobiológico. Necessita-se avançar na investigação a respeito dos processos que influenciam o temperamento, tanto na perspectiva biológica, com estudos genético-comportamentais, quanto na perspectiva contextual, com estudos transculturais.

\section{REFERÊNCIAS}

Blair, C. (2002). Early intervention for low birth weight, preterm infants: the role of negative emotionality in the specification of effects. Developmental Psychopathology, 14(2), 311-332.*

Bruce, J., Davis, E. P., \& Gunnar, M. R. (2002). Individual differences in children's cortisol response to the beginning of a new school year. Psychoneuroendocrinology, 27(6), 635-650."

Buss, A. H. \& Plomin, R. (1984). Temperament: early developing personality traits. Hillsdale, NJ: Erlbaum.

Chang, F. \& Burns, B. M. (2005). Attention in preschoolers: associations with effortful control and motivation. Child Development, 76(1), 247-263.

Chang, K. D., Blasey, C. M., Ketter, T. A., \& Steiner, H. (2003). Temperament characteristics of child and adolescent bipolar offspring. Journal of Affective Disorders, 77(1), 11-19.*

Couperus, J. W., \& Nelson, C. A. (2006). Early brain development and plasticity. In K. McCartney \& D. Phillips (Eds.). Blackwell handbook of early childhood development (pp. 85-105). Malden: Blackwell Publishing. 
Davis, E. P., Bruce, J., \& Gunnar, M. R. (2002). The anterior attention network: associations with temperament and neuroendocrine activity in 6-year-old children. Developmental Psychobiology, 40(1), 43-56."

Doussard-Roosevelt, J. A., Montgomery, L. A., \& Porges, S. W. (2003). Short-term stability of physiological measures in kindergarten children: respiratory sinus arrhythmia, heart period, and cortisol. Developmental Psychobiology, 43(3), 230-242."

Fox, N. A., Henderson, H. A., Rubin, K. H., Calkins, S., D., \& Schmidt, L. A. (2001). Continuity and discontinuity of behavioral inhibition and exuberance: Psychophysiological and behavioral influences across the first four years of life. Child Development, 72(1), 1-21.

Gleason, T. R., Gower, A. L., Hohmann, L. M., \& Gleason, T. C. (2005). Temperament and friendship in preschoolaged children. International Journal of Behavioral Development, 29(4), 336-344.

Goldsmith, H. H. (1983). Genetic influences on personality from infancy to adulthood. Child Development, 54(2), 331-355.

Goldsmith, H. H., Buss, A. H., Plomin, R., Rothbart, M. K., Thomas, A., Chess, S., et al. (1987). Roundtable: what is temperament? four approaches. Child Development, 58(2), 505-529.

Gorman, K. S., Lourie, A. E., \& Choudhury, N. (2001). Differential patterns of development: the interaction of birth weight, temperament, and maternal behavior. Developmental and Behavioral Pediatrics, 22(6), 366$375 .{ }^{*}$

Gunnar, M. R., Sebanc, A. M., Tout, K. Donzella, B., \& vanDulmen, M. M. H. (2003). Peer rejection, temperament, and cortisol activity in preschoolers. Developmental Psychobiology, 43(4), 346-358."

Henderson, H. A., Marshall, P. J., Fox, N., \& Rubin, K. H. (2004). Psychophysiological and behavioral evidence for varying forms and functions of nonsocial behavior in preschoolers. Child Development, 75(1), 236-250."

Ispa, J. M., Fine, M. A., \& Thornburg, K. R. (2002). Maternal personality as a moderator of relations between difficult infant temperament and attachment security in low-income families. Infant Mental Health Journal, 23(12), 130-144."

Kagan, J. (1998). Biology and the child. In W. Damon \& N. Eisenberg (Orgs.), Handbook of child psychology: Social, emotional, and personality development (vol. 3, pp. 177235). New York: Wiley.

Kagan, J., Reznick, J. S., \& Snidman, N. (1987). The physiology and psychology of behavioral inhibition in children. Child Development, 58, 1459-1473.

Kieras, J. E., Tobin, R. M., Graziano, W. G., \& Rothbart, M. K. (2005). You can't always get what you want: effortful control and children's responses to undesirable gifts. Psychological Science, 16(5), 391-396.

Klein, V. C. \& Linhares, M. B. M. (2007). Temperamento, comportamento e experiência dolorosa na trajetória de desenvolvimento da criança. Cadernos de Psicologia $e$ Educação - Paidéia, 17(36), 33-44.
Klein, V. C., Putnam, S. P., \& Linhares, M. B. M. (2009). Assessment of temperament in children: translation of instruments to Portuguese (Brazil) Language. Interamerican Journal of Psychology, 43(3), 442-447.

Kochanska, G., Gross, J. N., Lin, M. H., \& Nichols, K. E. (2002). Guilt in young children: Development, determinants, and relations with a broader system of standards. Child Development, 73(2), 461-482."

Luciana, M., Gunnar, M. R., Davis, E. P., Nelson, C. A., \& Donzella, B. (2005) Children's "catastrophic responses" to negative feedback on CANTAB's ID/ED set-shifting task: relation to indices of a depressive temperamental style. Cognitie Creier Comportament, 9(2), 343-361.*

Mervielde, I., de Clercq, B., de Fruyt, F., \& Van Leeuwen, K. (2006). Temperament, personality, and developmental psychopathology as childhood antecedents of personality disorders. Journal of Personality Disorders, 19(2), 171201.

Muris, P. \& Ollendick, T. H. (2005). The role of temperament in the etiology of child psychopathology. Clinical Child and Family Psychology Review, 8(4), 271289.

Putnam, S. P., Gartstein, M. A., \& Rothbart, M. K. (2006). Measurement of fine-grained aspects of toddler temperament: the Early Childhood Behavior Questionnaire. Infant Behavior and Development, 29(3), 386-401.

Ramos, M. C., Guerin, D. W., Gottfried, A. W., Bathurst, K., \& Oliver, P. H (2005). Family conflict and children's behavior problems: the moderating role of child temperament. Structural Equation Modeling, 12(2), 278298.

Rieser-Danner, L. A. (2003). Individual differences in infant fearfulness and cognitive performance: a testing, performance, or competence effect? Genetic, Social, and General Psychology Monographs. 129(1), 41-71.*

Rothbart, M. K. \& Bates, J. E. (1998). Temperament. In W. Damon \& N. Heisenberg (Orgs.), Handbook of Child Psychology, Social, emotional, and personality development . vol. 3 ( pp.105-176). New York: Wiley.

Rothbart, M. K. (1981). Measurement of temperament in infancy. Child Development, 52, 569-578.

Rubin, K. H., Burgess, K. B., \& Hastings, P. D. (2002). Stability and social-behavioral consequences of toddlers' inhibited temperament and parenting behaviors. Child Development, 73(2), 483-495.

Russell, A., Hart, C. H., Robinson, C. C., \& Olsen, S. F. (2003). Children's sociable and aggressive behavior with peers: a comparison of the US and Australian, and contributions of temperament and parenting styles. International Journal of Behavioral Development, 27(1), $74-86{ }^{*}$

Saylor, C. F., Boyce, G. C., \& Price, C. (2003). Early predictors of school-age behavior problems and social skills in children with intraventricular hemorrhage (IVH) and/or extremely low birthweight (ELBW). Child Psychiatry and Human Development, 33(3), 175-192." 
Schieche, M. \& Spangler, G. (2005). Individual differences in biobehavioral organization during problem-solving in toddlers: the influence of maternal behavior, infantmother attachment, and behavioral inhibition on the attachment-exploration balance. Developmental Psychobiology, 46(4), 293-306."

Spere, K. A., Schmidt, L. A., Theall-Honey, L. A., \& MartinChang, S. (2004). Expressive and receptive language skills of temperamentally shy preschoolers. Infant and Child Development, 13(2), 123-133.*

Szewczyk-Sokolowski, M., Bost, K. K., \& Wainwright, A. B. (2005). Attachment, temperament, and preschool children's peer acceptance. Social Development, 14(3), 379-397.

Thomas, A., \& Chess, S. (1977). Temperament and development. New York: Brunner/Mazel.

van Bakel, H. J. \& Riksen-Walraven, J. M. (2004). Stress reactivity in 15-month-old infants: links with infant temperament, cognitive competence, and attachment security. Developmental Psychobiology , 44(3), 157-167.

Volling, B. L., Herrera, C., \& Poris, M. P. (2004). Situational affect and temperament: Implications for sibling caregiving. Infant and Child Development, 13(2), 173-183."

Volling, B. L., McElwain, N. L., \& Miller, A. L. (2002). Emotion regulation in context: The jealousy complex between young siblings and its relations with child and family characteristics. Child Development, 73(2), 581$600{ }^{*}$
Watamura S. E., Donzella, B., Kertes, D. A., \& Gunnar, M. R. (2004). Developmental changes in baseline cortisol activity in early childhood: relations with napping and effortful control. Developmental Psychobiology, 45(3), $125-133 .^{*}$

Weinfield, N. S., Whaley, G. J., \& Egeland, B. (2004). Continuity, discontinuity, and coherence in attachment from infancy to late adolescence: sequelae of organization and disorganization. Attachment and Human Development, 6(1), 73-97."

Wolfe, C. D. \& Bell, M. A. (2004). Working memory and inhibitory control in early childhood: Contributions from physiology, temperament, and language. Developmental Psychobiology, 44(1), 68-83."

Zwaigenbaum, L., Bryson, S., Rogers, T., Roberts, W., Brian, J., \& Szatmari, P. (2005). Behavioral manifestations of autism in the first year of life. International Journal of Developmental Neuroscience, 23(2-3), 143-152.

Recebido em 10/12/2008 Aceito em 12/08/2010

\section{Endereço para correspondência:}

Maria Beatriz Martins Linhares. Laboratório de Pesquisa em Prevenção de Problemas de Desenvolvimento e Comportamento da Criança. Avenida Tenente Catão Roxo, 2650, Prédio da Saúde Mental (salas 52/ 53), Faculdade de Medicina de Ribeirão Preto-USP. Campus Monte Alegre-USP, CEP 14051-140, Ribeirão Preto-SP, Brasil. E-mail: linhares@ fmrp.usp.br. 